\section{(6) OPEN ACCESS}

\title{
Mapping genetic vulnerabilities reveals BTK as a novel therapeutic target in oesophageal cancer
}

\author{
Irene Yushing Chong, ${ }^{1}$ Lauren Aronson, ${ }^{1}$ Hanna Bryant, ${ }^{1}$ Aditi Gulati, ${ }^{1}$ \\ James Campbell, ${ }^{1}$ Richard Elliott, ${ }^{1}$ Stephen Pettitt, ${ }^{1}$ Paul Wilkerson, ${ }^{1}$ \\ Maryou B Lambros, ${ }^{1}$ Jorge S Reis-Filho, ${ }^{2}$ Anisha Ramessur, ${ }^{3}$ Michael Davidson, ${ }^{3}$ \\ Ian Chau, ${ }^{3}$ David Cunningham, ${ }^{3}$ Alan Ashworth, ${ }^{4}$ Christopher J Lord ${ }^{1}$
}

\begin{abstract}
- Additional material is published online only. To view please visit the journal online (http://dx.doi.org/10.1136 gutjnl-2017-314408).
\end{abstract}

${ }^{1} T$ The CRUK Gene Function Laboratory and Breast Cancer Now Toby Robins Breast Cancer Research Centre, The Institute of Cancer Research, London, UK ${ }^{2}$ Memorial Sloan Kettering Cancer Center, New York, NY, UK

${ }^{3}$ The Royal Marsden Hospital NHS Foundation Trust, London, UK

${ }^{4}$ UCSF Helen Diller Family Comprehensive Cancer Center, San Francisco, CA, USA

\section{Correspondence to} Dr Christopher J Lord, The CRUK Gene Function Laboratory and Breast Cancer Now Toby Robins Breast Cancer Research Centre, The Institute of Cancer Research, London, SW3 6JB, UK ; Chris.lord@icr.ac.uk

Received 27 April 2017 Revised 16 July 2017 Accepted 18 July 2017 Published Online First 22 August 2017
Check for updates

To cite: Chong IY, Aronson L, Bryant $\mathrm{H}$, et al. Gut 2018:67:1780-1792.

\section{ABSTRACT}

Objective Oesophageal cancer is the seventh most common cause of cancer-related death worldwide. Disease relapse is frequent and treatment options are limited.

Design To identify new biomarker-defined therapeutic approaches for patients with oesophageal cancer, we integrated the genomic profiles of 17 oesophageal tumour-derived cell lines with drug sensitivity data from small molecule inhibitor profiling, identifying drug sensitivity effects associated with cancer driver gene alterations. We also interrogated recently described RNA interference screen data for these tumour cell lines to identify candidate genetic dependencies or vulnerabilities that could be exploited as therapeutic targets.

Results By integrating the genomic features of oesophageal tumour cell lines with siRNA and drug screening data, we identified a series of candidate targets in oesophageal cancer, including a sensitivity to inhibition of the kinase BTK in MYC amplified oesophageal tumour cell lines. We found that this genetic dependency could be elicited with the clinical BTK/ERBB2 kinase inhibitor, ibrutinib. In both MYC and ERBB2 amplified tumour cells, ibrutinib downregulated ERK-mediated signal transduction, CMYC Ser-62 phosphorylation and levels of MYC protein, and elicited $\mathrm{G}_{1}$ cell cycle arrest and apoptosis, suggesting that this drug could be used to treat biomarker-selected groups of patients with oesophageal cancer.

Conclusions BTK represents a novel candidate therapeutic target in oesophageal cancer that can be targeted with ibrutinib. On the basis of this work, a proof-of-concept phase II clinical trial evaluating the efficacy of ibrutinib in patients with MYC and/or ERBB2 amplified advanced oesophageal cancer is currently underway (NCT02884453).

Trial registration number NCT02884453; Pre-results

\section{INTRODUCTION}

Oesophageal carcinoma is the seventh leading cause of cancer-related mortality and represents an area of unmet clinical need. ${ }^{1}$ Over 450000 people worldwide are affected with this disease, and the incidence of oesophageal adenocarcinoma (EAC) continues to rise rapidly, although oesophageal squamous cell carcinoma (ESCC) is the most common histology. ${ }^{2}$ Integrated genomic characterisation of oesophageal carcinoma has revealed
Significance of this study

What is already known on this subject?

- Oesophageal cancer is the seventh leading cause of cancer-related mortality and represents an area of unmet clinical need.

- Additional targeted approaches to treating this disease are urgently required, particularly in patients who progress after first-line therapy.

- Genomic profiling of oesophageal cancers has highlighted the presence of amplifications in oncogenes including CCND1, ERBB2 and $M Y C$, and efforts are required to exploit this information to identify therapeutic targets that could be used to treat biomarker-selected groups of patients with oesophageal cancer

What are the new findings?

- By analysing large-scale in vitro functional genomic profiling data and drug data from tumour cell models of oesophageal cancer and integrating this with genomic profiling of these models, we have identified a series of candidate genetic vulnerabilities associated with cancer driver gene alterations common in oesophageal cancers.

- One of the most profound effects identified was an addiction to the kinase BTK in MYC amplified oesophageal tumour cells, which could be elicited with the kinase inhibitor ibrutinib and which also targeted oesophageal tumour cells with ERBB2 amplification.

How might it impact on clinical practice in the foreseeable future?

- As the result of the observations made in this study, a proof-of-concept phase II clinical trial evaluating the efficacy of ibrutinib in patients with MYC and/or ERBB2 amplified advanced oesophageal cancer is currently underway (NCT02884453).

a separation between EAC and ESCC. This is demonstrated by a closer resemblance of genomic sequencing data from ESCC with squamous cell carcinomas of other anatomical sites than when compared with genomic profiles from primary EAC. ${ }^{3}$ EAC and ESCC are also distinct in terms 
of their aetiology in that EAC may originate from Barrett's oesophagus, a premalignant condition that arises as a complication of chronic gastro-oesophageal reflux disease, ${ }^{4}$ whereas ESCC does not. Overall, 5-year survival rates over the past 30 years have improved from 5\% to $19 \%$. However, the prognosis for patients diagnosed with oesophageal cancer remains poor. ${ }^{56}$ Patients with EAC frequently present with advanced, inoperable disease, but even in those patients with operable disease the frequency of disease recurrence after surgery remains high. ${ }^{2}$ Treatment options for patients with oesophageal cancers are extremely limited. Platinum-based chemotherapy remains the mainstay of treatment for EAC in the perioperative and metastatic setting, ${ }^{7-11}$ and although taxanes and camptothecin analogues have been introduced into treatment regimens, these have not delivered large improvements in median survival. ${ }^{12-14}$ A small number of targeted therapeutic approaches exist for patients with EAC; these include the antivascular endothelial growth factor receptor 2 (anti-VEGFR2) therapeutic antibody ramucirumab, used in patients who progress after fluoropyrimidine or platinum-containing chemotherapy, ${ }^{15}{ }^{16}$ and the Erb-B2 receptor tyrosine kinase (ERBB2) antibody, trastuzumab, used in combination with platinum-based chemotherapy as a firstline therapy in patients whose tumours exhibit ERBB2 oncogene amplification and/or overexpression. ${ }^{17}$ However, there are no licensed targeted therapies for patient with ESCC. Therefore, there is a clear need to identify additional targeted approaches to treating this disease, particularly in those patients who progress after first-line therapy.

One approach to this problem has been to define the molecular make-up of the disease, with the ultimate aim of using this information to inform the design of novel biomarker-stratified therapeutic approaches. For example, genomic profiling of oesophageal cancers has defined the mutational landscape of the disease. ${ }^{318-24}$ This work has highlighted the presence of a number of recurrent somatically occurring mutations in the disease, including amplifications of several well-known oncogenes including cyclin D1 (CCND1 15\% in $\mathrm{EAC}^{3}$ and 46\% in $\left.\operatorname{ESCC}^{23}\right), \operatorname{ERBB2}\left(32 \%\right.$ in $\mathrm{EAC}^{3}$ and up to $11 \%$ in $\left.\mathrm{ESCC}^{25}\right)$ and MYC (32\% in EAC and 23\% in ESCC $\left.{ }^{3}\right)$. Building on this work, efforts are now required to exploit this information to identify therapeutic targets that could be used to treat biomarker-selected groups of patients with oesophageal cancer. Here, we describe an analysis of large-scale in vitro functional genomic profiling of tumour cell models of oesophageal cancer. Integrating this functional profiling with genomic profiling of these models, we identify a series of candidate genetic vulnerabilities associated with cancer driver gene alterations common in oesophageal cancers. One of the most profound effects identified in this way was an addiction to the kinase BTK in MYC amplified oesophageal tumour cells; this addiction could be exploited with the kinase inhibitor ibrutinib, which also targeted oesophageal tumour cells with ERBB2 amplification, suggesting a novel candidate therapeutic approach in EAC and ESCC.

\section{RESULTS \\ Identification of genetic dependencies in oesophageal tumour cell lines that are associated with cancer driver gene alterations}

We aimed to identify genetic dependencies (GD) (ie, gene addiction and synthetic lethal effects) in oesophageal cancer that are associated with the presence of alterations in known oncogenic driver genes. To do this, we (1) compiled copy number and mutation profiles of 17 oesophageal tumour-derived cell lines using array comparative genomic hybridisation (aCGH) and exome sequencing data; (2) used these data to classify tumour cell lines according to the presence or absence of alterations in specific cancer driver genes, and then (3) interrogated recently described RNA interference (RNAi) screen data for the same tumour cell lines $^{26}$ to identify RNAi reagents that selectively targeted oesophageal tumour cell lines with cancer driver gene alterations. In addition, we carried out small molecule inhibitor drug sensitivity profiling in the same oesophageal tumour cell line panel, and by integrating these data with the genomic profiles of the tumour cell lines identified drug sensitivity effects associated with alterations in specific cancer driver genes (figure 1A).

\section{Genomic profiling of oesophageal tumour cell lines}

We selected 17 tumour cell lines, eight of which were derived from EAC (OE19, OE33, OACP4C, FLO-1, SK-GT-4, KYAE-1, ESO26 and JH-EsoAd1) and nine of which were derived from ESCC (KYSE70, TE1, TE5, TE6, TE8, TE9, TE10, TE11 and TE14; table 1). We used in-house aCGH data (32K based array CGHarray platform ${ }^{27}$ ) and publicly available single nucleotide polymorphism genotyping data (http://cancer.sanger.ac. uk/cosmic,https://portals.broadinstitute.org/ccle/home; online supplementary tables 1-17) to determine the copy number profile of each tumour cell line and used publicly available exome sequencing data to identify coding sequence variants in each model. We then cross-referred these tumour cell line genomic profiles to those from primary oesophageal tumours $21232428-34$ to annotate tumour cell lines in the panel according to the presence or absence of mutations in genes known to be recurrently mutated, amplified or deleted in primary oesophageal tumours. Cancer driver genes harbouring coding mutations or copy number alterations in two or more oesophageal tumour cell lines are illustrated in figure 1B and online supplementary table 18 .

Consistent with the mutational spectrum of the clinical disease, we found that mutations in genes such as TP53 (mutated in 72\% of primary EAC $^{19}$ and $83 \%$ of ESCC $^{23}$ ) were highly prevalent in the oesophageal tumour cell line panel. Six cell lines (OE19, OACP4C, SK-GT-4, KYSE70, TE9 and TE14) were predicted to have premature truncating TP53 mutations and 11 cell lines were predicted to have missense TP53 mutations (OE33, FLO-1, KYAE-1, ESO26, JHEsoAd1, TE1, TE5, TE6, TE8, TE10 and TE11). The SYNE1 gene (spectrin repeat containing nuclear envelope protein 1 gene), mutated in approximately $25 \%$ of primary treatment-naïve EAC tumours, ${ }^{19}$ was mutated in six oesophageal cell lines (FLO-1, KYAE-1, KYSE70, TE5, TE9 and TE10). Nine out of the 17 oesophageal tumour cell lines harboured homozygous copy number deletion of CDKN2A (cyclin-dependent kinase inhibitor $2 \mathrm{~A}, \mathrm{p} 16 / \mathrm{p} 14$ gene), found to be deleted in $76 \%$ of EAC and ESCC of the oesophagus. ${ }^{3}$ In addition, 35\% of the tumour cell line panel exhibited ERBB2 amplification events, a frequency comparable to the ERBB2 amplification rate in primary EACs. ${ }^{3}$ Furthermore, amplification of CCND1 (Cyclin D1 gene) was present in seven oesophageal models (JHEsoAd1, TE6, TE8, TE9, TE10, TE11 and TE14), and MYC amplification was present in five oesophageal cancer cell lines (OACP4C, KYAE-1, TE8, TE9 and TE10); in the clinical disease CCND1 amplification events are found in $15 \%$ of primary $\mathrm{EAC}^{3}$ and $46 \%$ of primary $\mathrm{ESCC}^{23}$ cases, whereas MYC is amplified in $23 \%$ in EAC and $32 \%$ in ESCC. ${ }^{3}$ In total, we identified 35 genes that were recurrently mutated in primary oesophageal tumours that were also mutated in at least 3 out of 17 oesophageal tumour cell line models. 
A

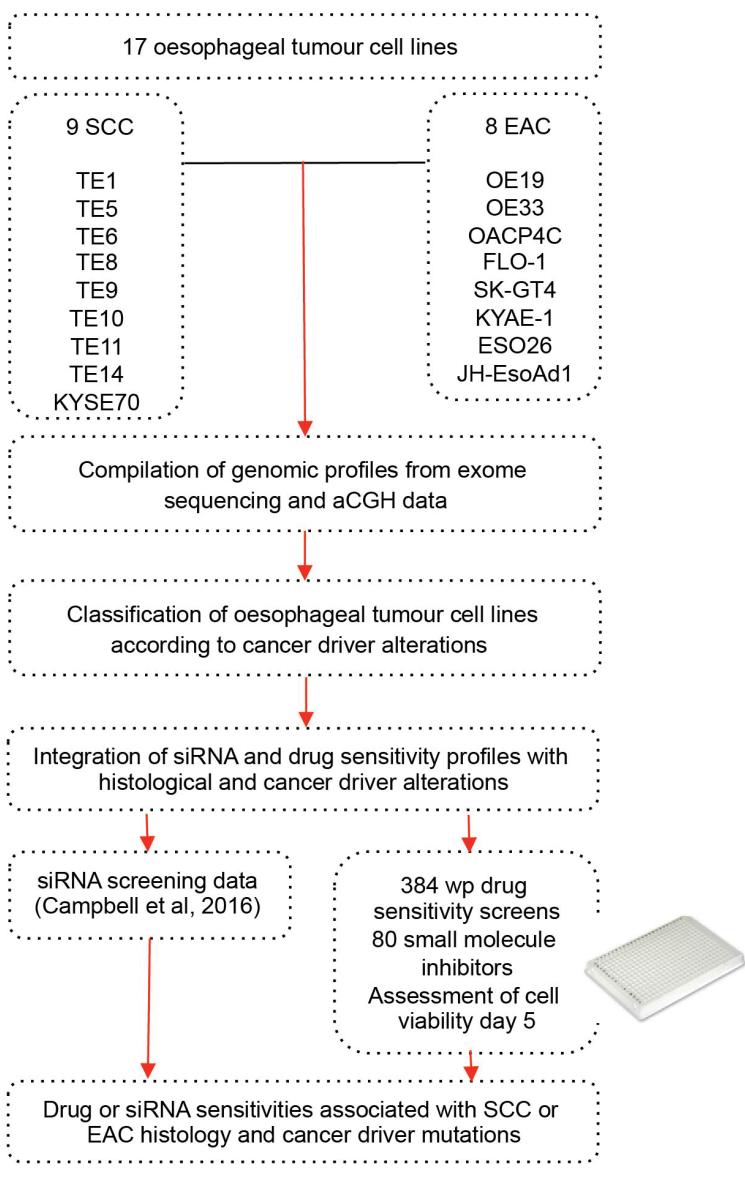

B

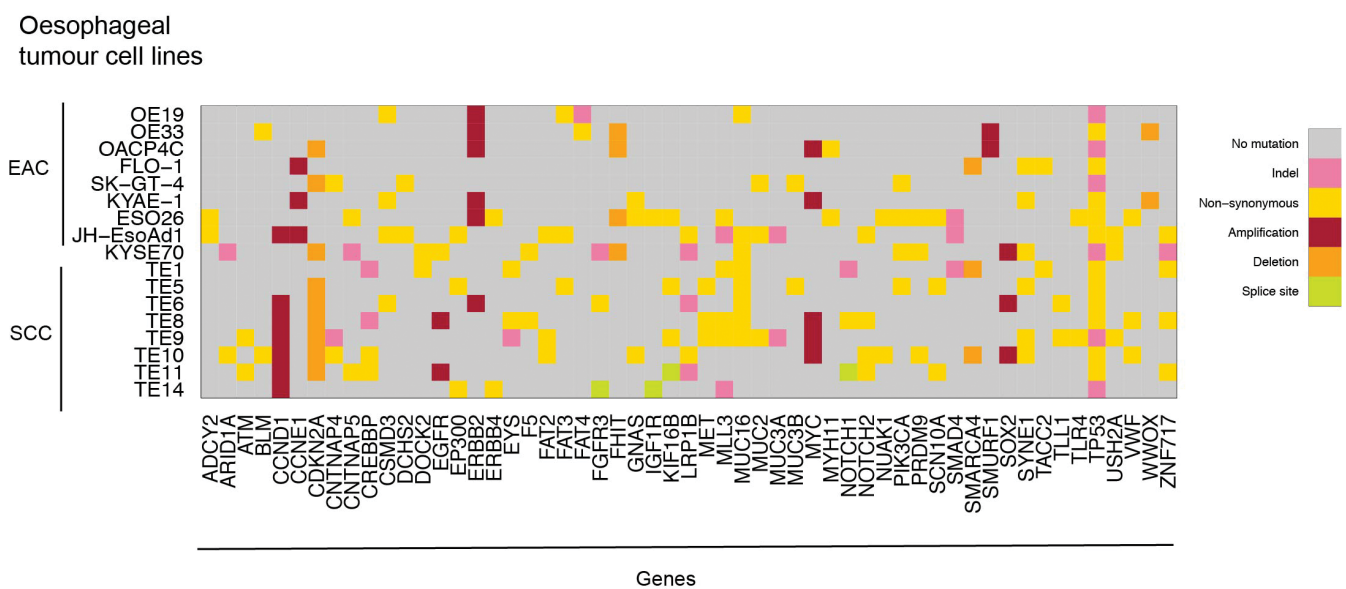

Figure 1 Identifying candidate genetic dependencies in oesophageal tumour cell lines. (A) Schematic of compilation of genomic profiles of 17 oesophageal tumour-derived cell lines using array comparative genomic hybridisation and exome sequencing. Oesophageal tumour cell lines were classified according to histology (SCC vs EAC) and cancer driver alterations. The 17 oesophageal cancer cell lines were screened in 384-well plate format using a small molecule drug library of compounds in late drug development or clinical trials. These drug data and high-throughput siRNA screening data (714 siRNA targeting kinase and kinase related genes ${ }^{26}$ ) from the same cell lines were interrogated and integrated with histological and cancer driver alterations to identify drug or siRNA sensitivities associated with SCC or EAC histology and cancer driver mutations. (B) Graph annotating predicted DNA coding mutations and copy number alterations present in two or more of 17 oesophageal cancer cell lines (8 EAC and $9 \mathrm{SCC}$ ) that are also to be found recurrently mutated in primary oesophageal tumours. aCGH, array comparative genomic hybridisation; EAC, oesophageal adenocarcinoma; SCC, squamous cell carcinoma.

\section{Small molecule drug sensitivity profiling and integration with} genomic profiles of oesophageal tumour cell lines

Using a 384-well plate screening approach, we assessed the sensitivity of each oesophageal tumour cell line to an in-house curated library of 80 drugs that are currently used for the treatment of cancer or which are in late-stage development (online supplementary table 19). Each drug in this library was present in four different concentrations (1, 10, 100 and $1000 \mathrm{nM})$. Each tumour cell line was plated in 384-well plates containing tissue culture media plus library small molecules and then cultured for 


\begin{tabular}{ll}
\hline $\begin{array}{l}\text { Table } 1 \text { Oesophageal tumour cell lines used for high-throughput } \\
\text { siRNA and drug screening }\end{array}$ \\
\hline Cell line & Histology \\
\hline OE19 & EAC \\
OE33 & EAC \\
OACP4C & EAC \\
\hline FL0-1 & EAC \\
\hline SK-GT4 & EAC \\
\hline KYAE-1 & EAC \\
\hline ES026 & EAC \\
\hline JH-EsoAd1 & EAC \\
\hline KYSE70 & SCC \\
\hline TE1 & SCC \\
\hline TE5 & SCC \\
\hline TE6 & SCC \\
\hline TE8 & SCC \\
\hline TE9 & SCC \\
\hline TE10 & SCC \\
\hline TE11 & SCC \\
\hline TE14 & SCC \\
\hline
\end{tabular}

EAC, oesophageal adenocarcinoma; SCC, squamous cell carcinoma.

a further 5 days, at which point cell viability was estimated using CellTiter-Glo reagent (figure 2A). Each tumour cell line was screened in triplicate, with 15 of the 17 tumour cell lines generating highly reproducible data (median correlation coefficients of $>0.9$ between replicates; online supplementary figure 1 ) and suitable dynamic range quality control criteria (estimated by $Z^{\prime}$ calculations). The cell viability measurements for each drug titration (online supplementary table 19) were used to generate area under the curve (AUC) dose response data for each tumour cell line, illustrated as a heatmap in figure $2 \mathrm{~A}$ and in online supplementary table 20 .

We found the vast majority of oesophageal tumour cell lines to be sensitive to conventional cytotoxic agents including gemcitabine, camptothecin and paclitaxel, as well as the HSP90 inhibitor 17-AAG, the polo-like kinase 1 (PLK1) inhibitor BI2536, the cyclin-dependent kinase modulator flavopiridol and the P21 activated kinase 4 inhibitor, PF03758309 (figure 2A and online supplementary table 20). We then assessed if drug sensitivity effects differed according to whether tumour cell lines were derived from EAC or ESCC (online supplementary table 21) and found that ESCC cell lines were more sensitive to four drugs including dasatinib $(\mathrm{p}=0.032$, median permutation (MP), t-test, figure 2B), a multitarget tyrosine kinase inhibitor and Src family tyrosine kinase inhibitor, and BI2536 ( $p=0.033$, MP test, figure 2C), a small molecule inhibitor of PLK1. Compared with EAC cell lines, the ESCC cell lines were more sensitive to the epidermal growth factor receptor (EGFR) small molecule inhibitor, gefitinib ( $p=0.034$, MP test, figure $2 \mathrm{D})$, a finding that was consistent with data from a subgroup analysis of the cancer oesophagus gefitnib (COG) trial that revealed an HR in favour of gefitinib treatment in patients with ESCC. ${ }^{35}$ In addition, ESCC cell lines were found to be more sensitive to the pan-ErbB inhibitor, canertinib ( $p=0.038$, MP test, figure $2 \mathrm{E})$, compared with EAC cell lines.

We integrated the data from the small molecule inhibitor screen with the genomic profiles of the tumour cell line panel to identify drug sensitivity effects associated with likely cancer driver gene alterations (figure 1A, online supplementary table 22). As expected, this analysis indicated that ERBB2 or EGFR amplified tumour cell lines were preferentially sensitive to the Erb-family kinase inhibitor lapatinib $(p=0.038$, Mann-Whitney $U$ test, figure $2 \mathrm{~F}$ ), providing proof of principle that this approach could identify drug sensitivities associated with molecularly defined subgroups of oesophageal cancer. We also noted that oesophageal tumour cell lines that harbour missense mutations predicted to result in amino acid changes in the PIK3CA helicase domain exhibited the greatest sensitivity to the PI3-kinase/AKT signalling pathway inhibitor BEZ235 ( $p=0.018$, MP test, figure $2 \mathrm{G}$ ). Oesophageal tumour cell lines harbouring mutations within the PIK3CA helicase domain also exhibited preferential sensitivity to the 3-phosphoinositide-dependent protein kinase 1 inhibitor GSK-2334470A ( $\mathrm{p}=0.021$, MP test $)$ and vinorelbine $(\mathrm{p}=0.032$, $\mathrm{MP}$ test, figure $2 \mathrm{H})$.

\section{Integration of genomic profiles with siRNA data}

Using a similar data integration scheme as for the drug sensitivity data, we cross-referred the genomic profiles of the tumour cell line panel to in-house generated data describing the sensitivity of each of the 17 oesophageal tumour cell line models and an additional 103 tumour cell lines to siRNAs designed to target a panel of 714 genes encoding kinases and kinase-related proteins (the 'Campbell et al data set' ${ }^{26}$ ) as a means to identify candidate GDs in oesophageal cancers (online supplementary figure 2 and supplementary table 23). As kinases represent a pharmacologically tractable family of enzymes, we reasoned that doing so might also identify targetable vulnerabilities associated with molecularly defined subsets of the disease (figure 1A).

Initially, we used this integrated analysis to identify those kinases that when targeted with siRNA selectively targeted the oesophageal tumour cell lines, as opposed to the other 103 tumour cell lines that we also profiled by siRNA screening in our data set. ${ }^{26}$ This analysis suggested that, compared with non-oesophageal tumour cell lines, those derived from oesophageal cancers exhibited dependencies on NEK9 $(\mathrm{p}=0.00026$, MP test), PACE1 ( $\mathrm{p}=0.00027, \mathrm{MP}$ test), MGC42105 ( $\mathrm{p}=0.0003$, $\mathrm{MP}$ test) and CDKN2C ( $\mathrm{p}=0.00058$, MP test) (figure $3 \mathrm{~A}$ and online supplementary table 24). When ESCC-derived cell lines were compared with EAC-derived cell lines, we found EGFR to be the most profound GD associated with ESCC histology $(\mathrm{p}=0.000006, \mathrm{MP}$ test, figure $3 \mathrm{~B}$ and online supplementary table 25). This observation confirms the enhanced sensitivity of ESCC cell lines to the EGFR small molecule inhibitor, gefitinib, and the pan-ErbB inhibitor, canertinib, seen in the small molecule drug screen (figure 2E). When using the genomic profiles of the oesophageal tumour cell lines to identify GD associated with alterations in specific cancer driver genes (online supplementary table 26), we found that, as expected, siRNA designed to target $E R B B 2$ itself preferentially targeted $E R B B 2$ amplified oesophageal tumour cell lines $(\mathrm{p}=0.019$, MP test, figure $3 \mathrm{D}, \mathrm{E})$, supporting the hypothesis that ERBB2 amplification can lead to an oncogene addiction effect. ${ }^{17}$ We found that ERBB2 amplified oesophageal tumour cell lines also exhibited GDs on members of the mitogen-activated protein kinase family that signal downstream of ERBB2 ${ }^{36}$ including MAPK2K2 ( $\mathrm{p}=0.0073$, MP test, figure $3 \mathrm{D}), M A P 2 K 3(\mathrm{p}=0.0082, \mathrm{MP}$ test, figure $3 \mathrm{~F})$ and MAPK6 $(\mathrm{p}=0.0082$, MP test, figure 3D).

MYC is amplified in 23\% of EAC and 32\% of ESCC. ${ }^{3}$ We found MYC amplified oesophageal tumour cell lines to exhibit GDs on CLK1 (Cdc2-like kinase gene, $\mathrm{p}=0.02$, MP test, figure $3 \mathrm{G}$ ), BTK (Bruton agammaglobulinaemia tyrosine kinase gene, $\mathrm{p}=0.017 \mathrm{MP}$ test, figure $3 \mathrm{H}$ ), ALK (anaplastic 
A

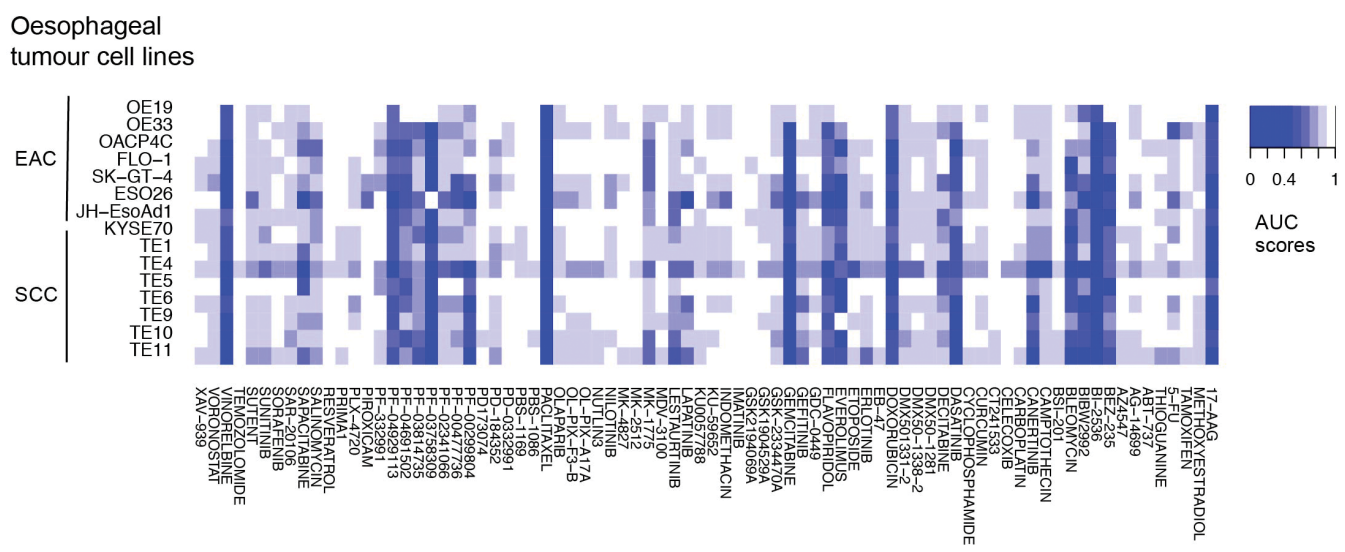

B

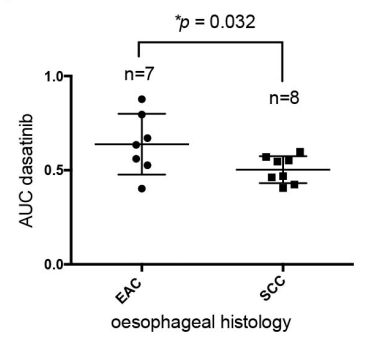

E

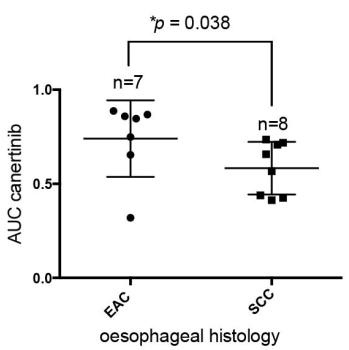

H

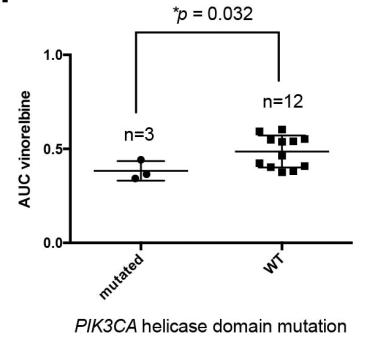

C

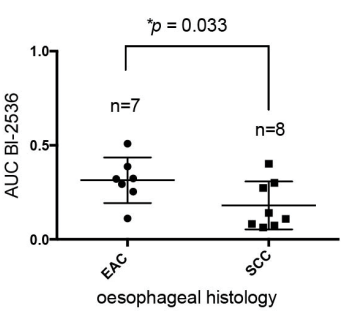

$\mathbf{F}$

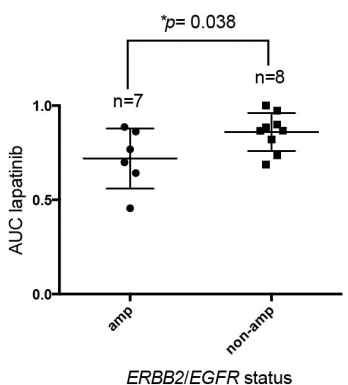

D

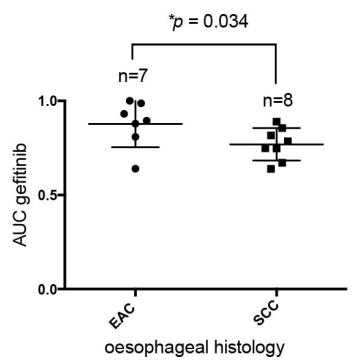

G

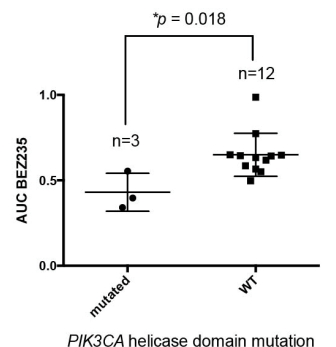

lymphoma receptor tyrosine kinase gene, $\mathrm{p}=0.008 \mathrm{MP}$ test, figure 3I) and PRKCA (protein kinase $\mathrm{C}$ alpha gene, $\mathrm{p}=0.028 \mathrm{MP}$ test, figure $3 \mathrm{G}$ ). Among other GDs identified in this way, we observed a relationship between SMARCA4 copy number loss and a GD on BRD4 ( $\mathrm{p}=0.022$, MP test, figure $3 \mathrm{~J}, \mathrm{~K}$ ), a member of the BET (bromodomain and extra terminal domain) family. CCND1 amplified oesophageal tumour cell lines exhibited GD on TYK2 (tyrosine kinase 2) tyrosine kinases (figure 3L). Other GDs identified in the CCND1 amplified oesophageal tumour cell lines included FLT1 (which encodes VEGFR1, $\mathrm{p}=0.0054$, MP test), ROCK1 (rho-associated, coiled-coil-containing protein kinase gene, 
A
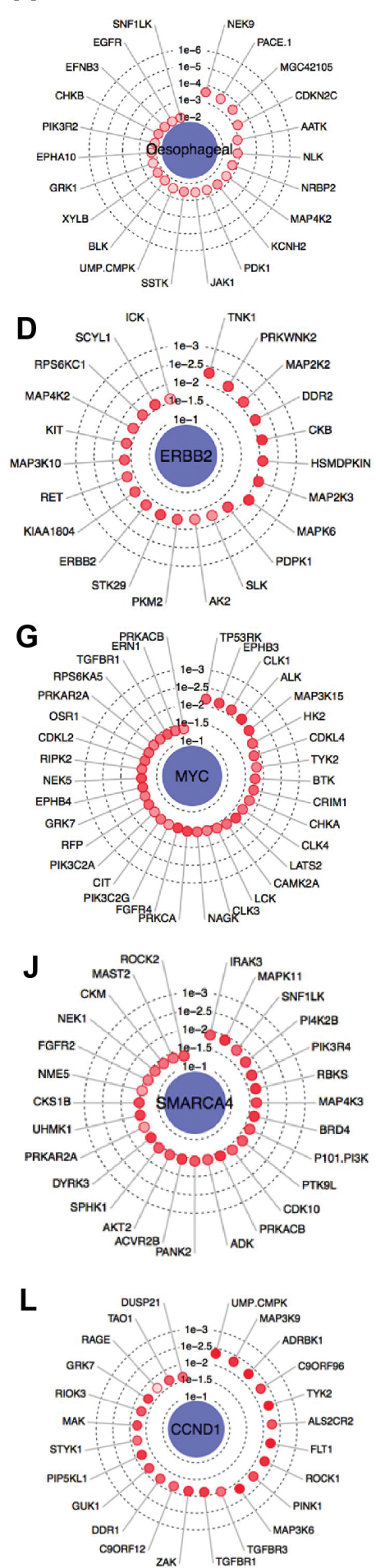

B
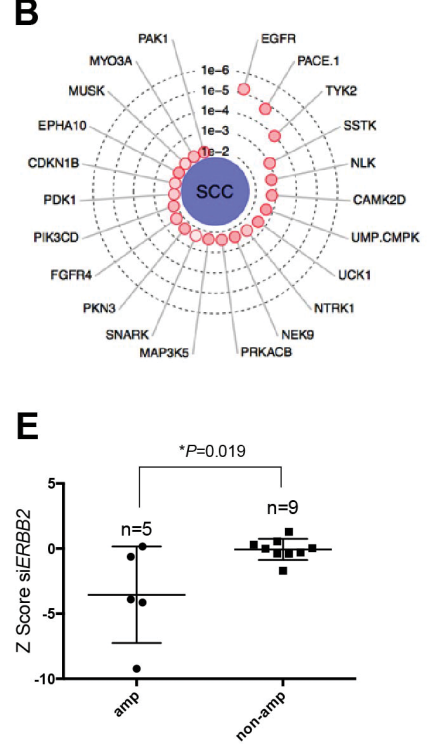

ERBB2 amplification status

H

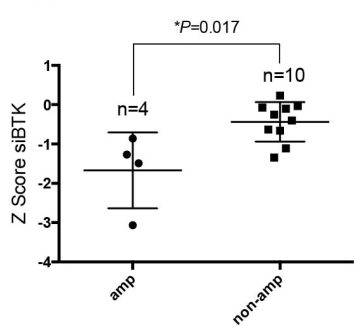

MYC amplification status

K
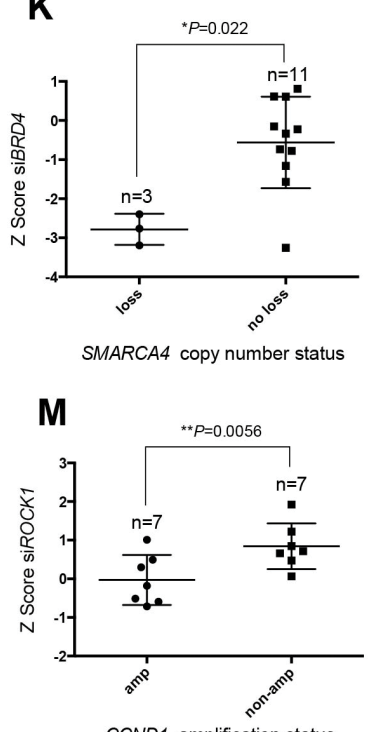

C

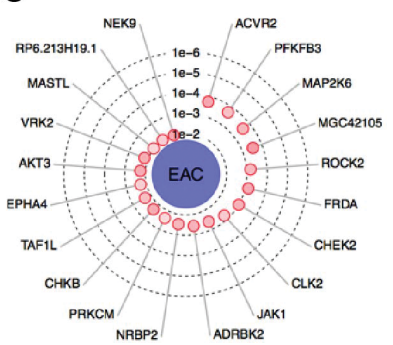

F

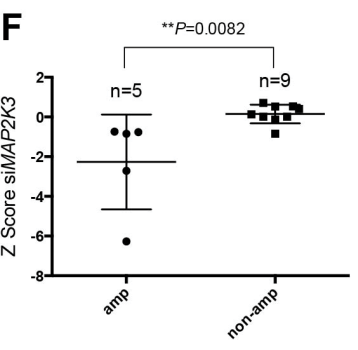

ERBB2 amplification status

I

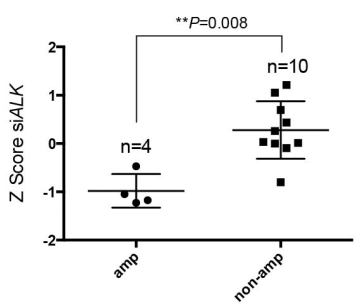

c-MYC amplification status

Figure 3 Integration of genomic profiles with siRNA data. (A) Radar plot showing key genetic dependencies associated with oesophageal tumour cell lines compared with tumour cell lines from other histologies. ${ }^{26}$ The concentric circles indicate the degree of statistical significance and depth of colour indicates separation of Z scores. Radar plots showing key genetic dependencies associated with (B) oesophageal SCC histology and (C) EAC histology. (D) Radar plot showing key genetic dependencies associated with ERBB2 amplification. Box and whisker plots of $Z$ score values showing that targeting of (E) ERBB2 ( $\mathrm{p}=0.019$, MP test) and (F) MAP2K3 ( $\mathrm{p}=0.0082$, MP test) is selectively lethal in ERBB2 amplified oesophageal cancer cell lines. (G) Radar plot showing key genetic dependencies associated with MYC amplification. $(\mathrm{H})$ Box and whisker plots of $Z$ score values showing that targeting of BTK ( $p=0.017$, MP test) and (I) ALK ( $p=0.008$, MP test) is selectively lethal in MYC amplified oesophageal cell lines. (J) Radar plot showing key genetic dependencies associated with copy number deletions in SMARCA4 in oesophageal cancer cell lines. (K) Box and whisker plots of Z score values showing that targeting BRD4 ( $p=0.022$, MP test) is selectively lethal in oesophageal tumour cell lines harbouring SMARCA4 copy number loss. (L) Radar plot showing key genetic dependencies associated with CCND1 amplification. (M) Box and whisker plots of Z score values showing that targeting ROCK1 ( $p=0.0056$, MP test) is selectively lethal in CCND1 amplified oesophageal cancer cell lines. EAC, oesophageal adenocarcinoma; MP, median permutation; SCC, squamous cell carcinoma. 
$\mathrm{p}=0.0056 \mathrm{MP}$ test, figure $3 \mathrm{M}$ ) and TGFBR1 (TGF $\beta$ receptor 1 gene, $\mathrm{p}=0.013$, MP test, figure $3 \mathrm{~L}$ ).

\section{Ibrutinib sensitivity in MYC and ERBB2 amplified oesophageal cancer}

Analysis of the oesophageal kinase dependency profiles revealed that one of the most profound associations was between MYC amplification and sensitivity to BTK siRNA ( $p=0.017$, MP test, figure $3 \mathrm{H}$ ). Although BTK has been most studied within the context of $\mathrm{B}$ cell malignancies ${ }^{37}$ and comprises a key component downstream of BCR signalling, ${ }^{38}$ BTK isoforms are also present in epithelial tumours including breast, ${ }^{39}$ prostate, ${ }^{40}$ colon $^{41}$ and gastric cancers. ${ }^{42}$ We assessed BTK protein and mRNA expression across the panel of oesophageal tumour cell lines and found that canonical BTK is expressed in these models, although at low levels (figure 4A,B). We also confirmed that a splice variant, BTK-C, is expressed in the oesophageal tumour models (figure 4C,D).

We validated the BTK/MYC synthetic lethal interaction in MYC amplified oesophageal tumour cell lines using multiple, different BTK-targeting siRNAs (figure 4E,F,G), suggesting that this was unlikely to be an off-target effect. In addition, we observed decreased cell viability (figure $4 \mathrm{H}$ ) and a reduction of BTKC mRNA expression after transfection with individual siRNAs specifically targeting BTK-C (figure $4 \mathrm{I}$ and online supplementary figure 3), suggesting that the BTK-C isoform is essential in oesophageal tumour models. To investigate whether small molecule inhibition of BTK could mimic these effects, we exposed the panel of oesophageal tumour cell lines to increasing concentrations $(0.1-10 \mu \mathrm{M})$ of the clinical BTK inhibitor ibrutinib, a drug that has durable single-agent efficacy in relapsed mantle cell lymphoma, Waldenstrom's macroglobulinaemia and chronic lymphocytic leukaemia. ${ }^{43}{ }^{44}$ While ibrutinib is a potent BTK inhibitor, it also directly inhibits ERBB2. ${ }^{45}{ }^{46}$ Consistent with this, our analysis of ibrutinib sensitivity in oesophageal tumour cell lines revealed that there was preferential sensitivity to ibrutinib in both MYC amplified and/or ERBB2 amplified oesophageal tumour cell lines $(\mathrm{p}=0.018$, Mann-Whitney U test, figure $5 \mathrm{~A}, \mathrm{~B})$.

We wanted to examine the mechanism of ibrutinib sensitivity in MYC amplified oesophageal cell lines. We found that MYC protein levels were reduced by ibrutinib exposure in both KYAE-1 (MYC amplified) and TE8 (MYC and ERBB2 amplified) oesophageal tumour cell lines (figure 5C,D). Transfection of siRNA targeting MYC confirmed that these tumour cell lines were addicted to MYC (figure 5E,F). Ibrutinib exposure also caused an increased $\mathrm{G}_{1}$ fraction in $M Y C$ amplified and $M Y C /$ ERBB2 coamplified oesophageal tumour cell lines (figure $5 \mathrm{G}, \mathrm{H}$ ) but not in tumour cell lines without these amplification events (figure 5I). In MYC amplified and also MYC/ERBB2 coamplified oesophageal tumour cell lines, ibrutinib exposure caused a reduction in phosphorylated $\mathrm{Rb}$, total $\mathrm{Rb}$ and cyclin $\mathrm{D} 1$ protein levels (figure $5 \mathrm{~J}, \mathrm{~K}$ ) not seen in non-amplified tumour cell lines (figure $5 \mathrm{~L}$ ), consistent with the increased $\mathrm{G}_{1}$ fraction. Ibrutinib exposure also increased annexin V and cleaved PARP1 levels in sensitive tumour cells (figure $5 \mathrm{M}, \mathrm{N}$ ), suggesting that the $\mathrm{G}_{1}$ arrest caused by ibrutinib might eventually drive an apoptotic response.

\section{ERK1/2 is downregulated by ibrutinib}

BTK is known to signal via the ras/mitogen-activated protein kinase (RAS-RAF-MEK-ERK) pathway. ${ }^{41}$ To investigate whether this contributed to the ibrutinib-dependent reduction of MYC protein levels in oesophageal tumour cells, we evaluated changes in $\mathrm{p}$-ERK protein expression after ibrutinib exposure. We observed a reduction in p-BTK (figure 6A), indicating decreased catalytic activity of BTK and p-ERK levels (figure 6B,C) after ibrutinib exposure. As ERK is a known mediator of MYC phosphorylation at serine $62,{ }^{47}$ we assessed changes in p-MYC S62 protein expression after exposure to ibrutinib and observed a decrease in p-MYC S62 levels in oesophageal tumour cell lines that were either MYC amplified or MYC/ERBB2 coamplified (figure 6D,E). Phosphorylated MYC at serine 62 (p-MYC S62) is required for stabilisation of MYC protein levels. ${ }^{48}$ Consistent with this, we observed a reduction in MYC protein levels (figure 6D,E). Taken together, these results suggested that exposure to ibrutinib resulted in a reduction in MYC in oesophageal tumour models by reduction of BTK-dependent, ERK-mediated, MYC phosphorylation.

\section{DISCUSSION}

In this study, we show how integration of functional genomic profiling data with genotypic data from oesophageal cancer tumour cell lines may be used to identify candidate GDs. By undertaking high-throughput small molecule drug screening, we provide a public resource for the identification of biomarker driven therapies in a disease that currently has very limited treatment options.

By interrogating drug and siRNA data with copy number profiling data, we identified an addiction to BTK in MYC amplified oesophageal tumour cell line models that may represent an induced essentiality where the gain of MYC function induces a dependency on BTK. ${ }^{49}$ We exploited this addiction with the small molecule inhibitor ibrutinib that targets both BTK and ERBB2, and found increased sensitivity in MYC and/or ERBB2 amplified oesophageal tumour models to ibrutinib. Ibrutinib exposure also resulted in downregulation of cMYC. Mechanistically, one model that is consistent with these data might be that BTK and ERBB2 inhibition with ibrutinib results in downregulation of cMYC, likely via deregulation of ERK signalling. The use of ibrutinib in this context exemplifies how molecular and functional profiling data might be used to design novel therapeutic approaches for patients with oesophageal cancer.

Ibrutinib already has Food and Drug Administration approval for the treatment of patients with Waldenstrom's macroglobulinaemia, ${ }^{50}$ mantle cell lymphoma ${ }^{44}$ and chronic lymphocytic leukaemia ${ }^{43}$ based on the demonstration of durable responses in single-arm multicentre clinical studies that enrolled patients with previously treated disease. Considering the known safety profile of ibrutinib, we have initiated a proof-of-concept single-arm phase II trial of ibrutinib in patients with advanced oesophagogastric cancer harbouring MYC and/or ERBB2 amplifications (ibrutinib in MYC amplified oesophageal cancer (iMYC)trial - NCT NCT02884453) who have progressed after first-line treatment. Patients recruited to the iMYC trial undergo prescreening for MYC and ERBB2 amplification by fluoresence in situ hybridisation criteria. The role for ibrutinib in ERBB2 amplified EAC may be clinically relevant as there are currently no approved anti-ERBB2 therapies in the second-line setting; approximately half of patients with advanced EAC have de novo resistance to first-line trastuzumab, and the median time to progression following initial response to chemotherapy and trastuzumab is only 7.1 months. ${ }^{17}$ Exploratory molecular and functional imaging biomarker analyses have been incorporated into the iMYC trial where serial tumour biopsies and blood samples are retrieved at screening, during treatment and 
A

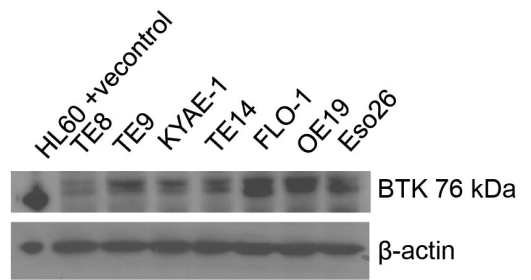

C

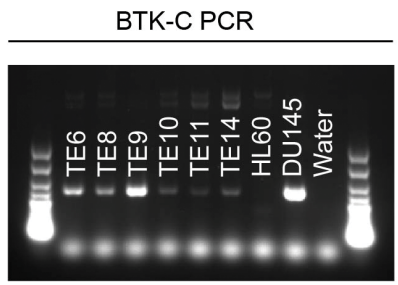

B

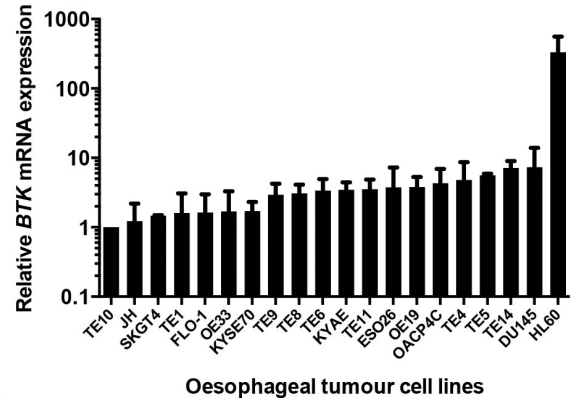

D

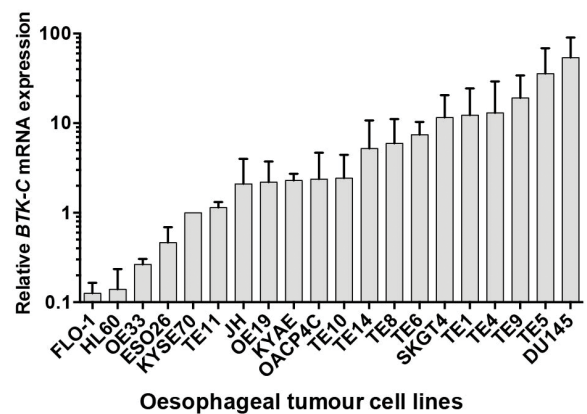

E

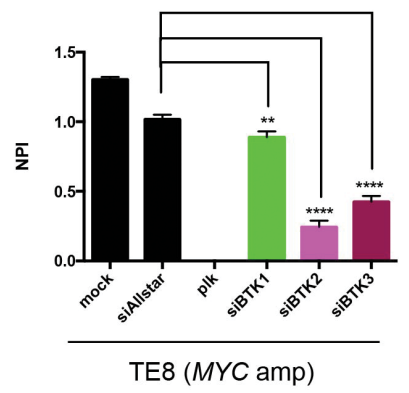

H

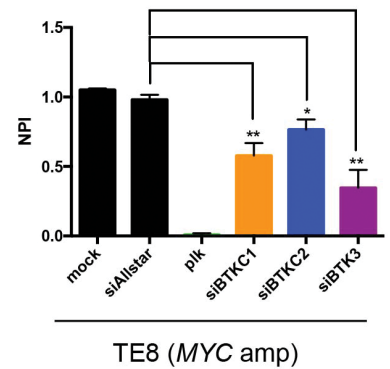

$\mathbf{F}$

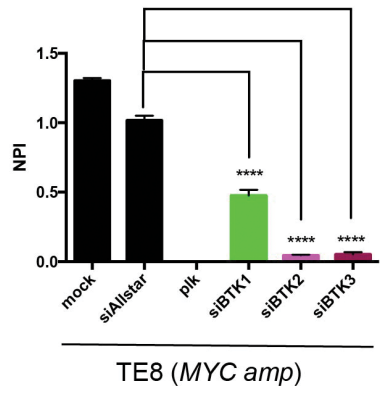

I

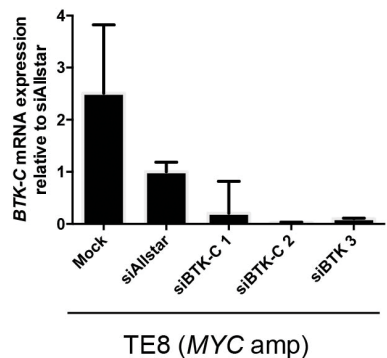

G

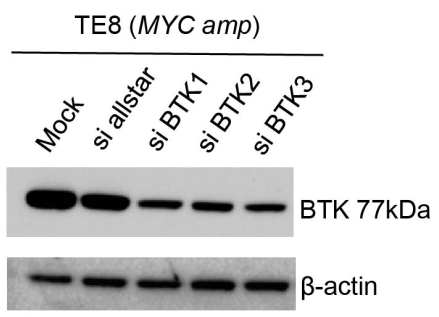

at progression. Whole exome sequencing and RNA sequencing of serial tumour samples will be undertaken to assess genetic determinants of response and resistance to ibrutinib. Sequential tumour samples will be evaluated for expression of phosphorylated kinases in the ERBB2, AKT and RAS-RAF-MEK-ERK-MYC signalling pathways as part of pharmacodynamic biomarker assessment. In addition, we will assess the expression of phosphorylated proteins that signal downstream of BTK, including 1-phosphatidylinositol-4,5-bisphosphonate phosphodiesterase gamma and protein kinase C. Given that we observed greatest 

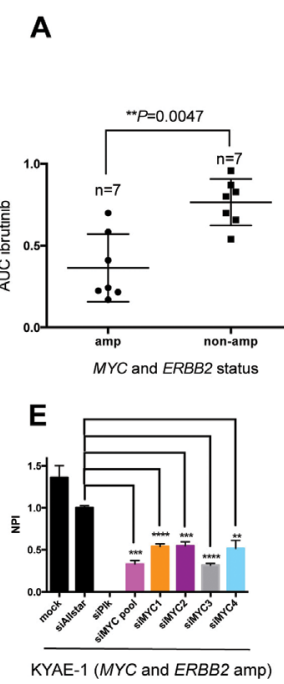

H

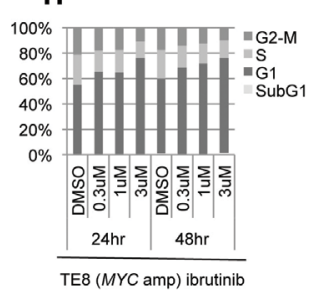

$\mathbf{K}$

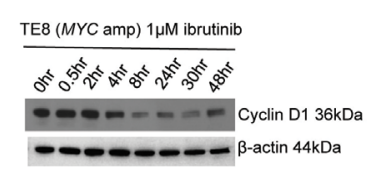

N

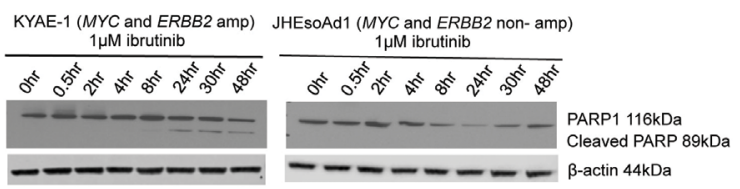

B

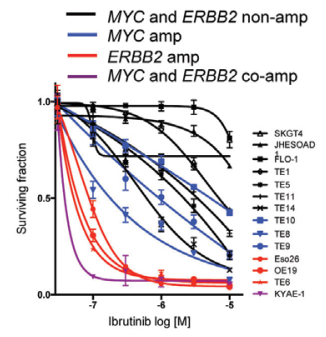

$\mathbf{F}$

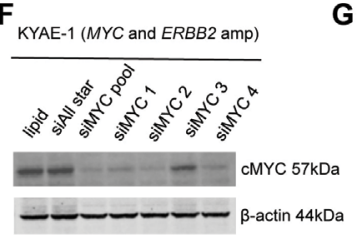

\section{I}

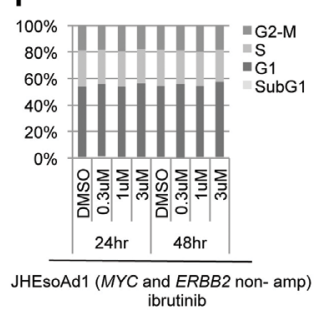

L JHEsoAd1 (MYC and ERBB2 non- amp)

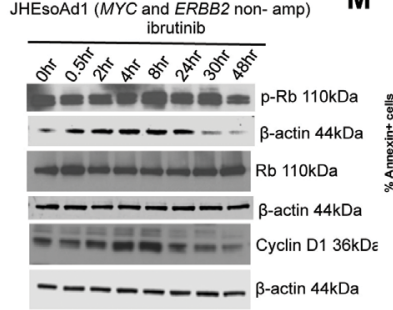

C KYAE-1 (MYC and ERBB2 amp)

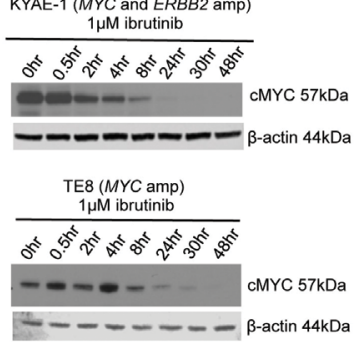

G

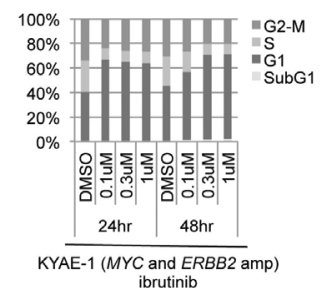

J
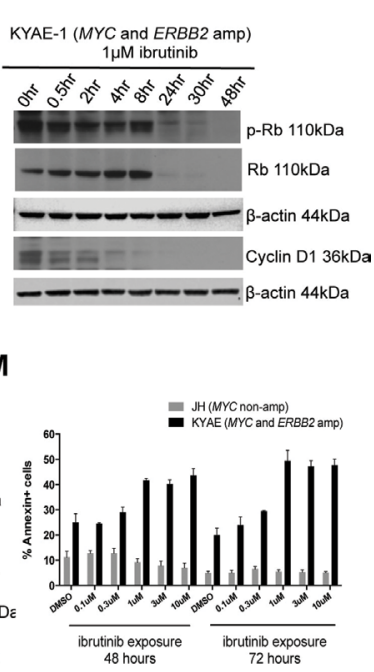

Figure 5 Ibrutinib sensitivity in MYC and/or HER2 amplified oesophageal tumour cell lines. (A) Box plot depicting area under the curve values for ibrutinib. Preferential sensitivity to ibrutinib is observed in oesophageal cancer cell lines harbouring $M Y C$ and/or ERBB2 amplification. (B) Ibrutinib drug sensitivity curves across the panel of oesophageal tumour cell lines. Cell lines that are not MYC or ERBB2 amplified are depicted in black, MYC and ERBB2 amplified in purple, MYC amplified alone in blue and ERBB2 amplified alone in red. (C) Western blots showing decreased expression of CMYC in MYC amplified KYAE-1 tumour cell line after exposure to $1 \mu \mathrm{M}$ ibrutinib. (D) Western blot showing decreased expression of CMYC in MYC amplified TE8 tumour cell line after exposure to $1 \mu \mathrm{M}$ ibrutinib. (E) Bar chart showing decreased cell viability in KYAE-1 (MYC and ERBB2 amplified) following transfection with siMYC oligonucleotides. (F) Western blot showing decreased protein expression of cMYC following transfection of siMYC oligonucleotides in KYAE-1 (MYC and ERBB2 amplified). (G) Cell cycle analysis after 24 and 48 hours of ibrutinib exposure in KYAE-1 (MYC and ERBB2 amplified), (H) TE8 (MYC amplified) and (I) JHEsoAd1 (MYC and ERBB2 non-amplified) tumour cell lines. Prolongation of the $\mathrm{G}_{1}$ phase is observed with increasing doses of ibrutinib in cell line harbouring MYC amplification. $\mathrm{G}_{1}$ arrest was not observed in JHEsoAd1 (MYC and ERBB2 non-amplified) cell line. (J) Western blot showing decreased expression of $\mathrm{p}-\mathrm{Rb}$, Rb and cyclin D1 following ibrutinib exposure in KYAE-1 (MYC and ERBB2 amplified). (K) Western blot showing decreased expression of cyclin D1 following ibrutinib exposure in TE8 (MYC amplified). (L) Western blot showing no change in protein expression of $\mathrm{p}-\mathrm{Rb}, \mathrm{Rb}$ and cyclin $\mathrm{D} 1$ following ibrutinib exposure in JHEsoAd1 (MYC and ERBB2 non amplified). (M) Bar chart showing the apoptotic cell fraction (percentage of annexin-positive cells) after exposure to increasing doses of ibrutinib (24 and 48 hours). An increase in apoptosis is observed in KYAE-1 (MYC and ERBB2 amplified) that is not seen in JHEsoAd1 (MYC and ERBB2 non-amplified). (N) Western blot showing expression of cleaved PARP1 after ibrutinib exposure in the KYAE-1 (MYC and ERBB2 amplified). Cleaved PARP1 is not seen in JHEsoAd1 after ibrutinib exposure (MYC and ERBB2 non-amplified).

sensitivity to ibrutinib in MYC/ERBB2 coamplified models (4\% of $\mathrm{EAC}^{3}$ ) and that ERBB2 amplification only occurs in up to $11 \%$ of ESCC $^{25}$ compared with up to $32 \%$ in EAC, ${ }^{3}$ it is likely that a therapeutic approach utilising ibrutinib will be clinically relevant to a larger proportion of patients with EAC within the context of this study.

Our aim in providing these data and illustrating its potential utility is to present testable hypotheses for future work that 
A

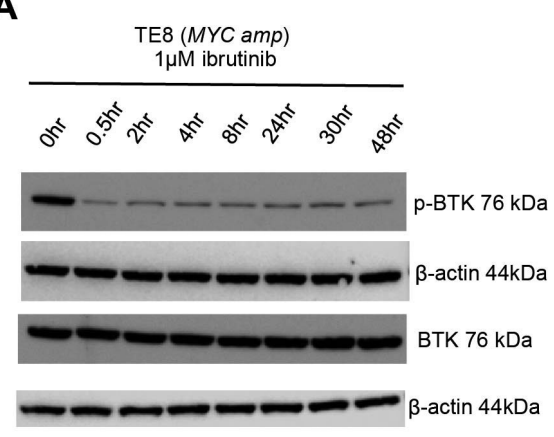

C

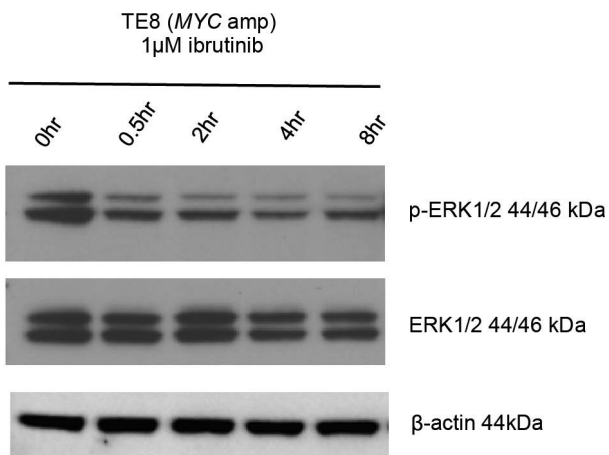

B

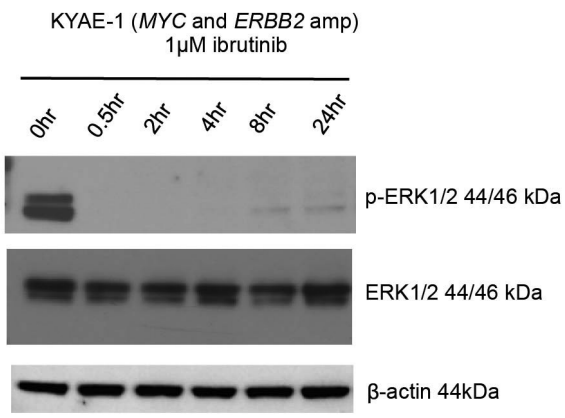

D

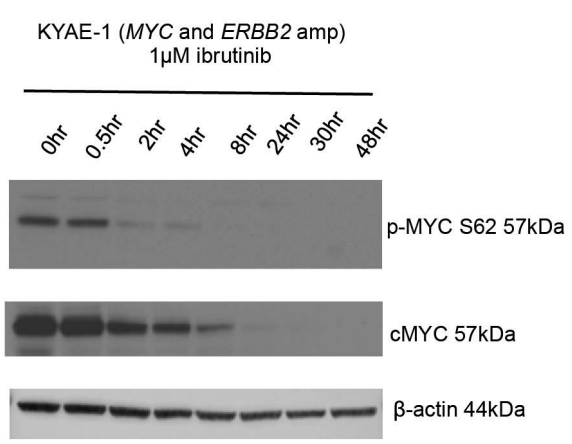

$\mathbf{E}$

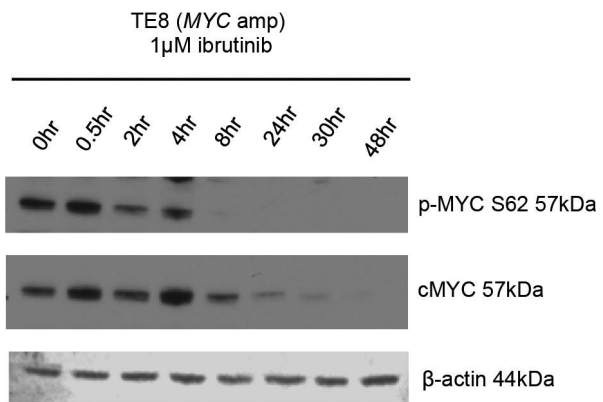

Figure 6 ERK1/2 is downregulated by ibrutinib. (A) Western blot showing decreased p-BTK protein expression in BTK overexpressed (lentiviral transduction) TE8 cell line (MYC amplified) following ibrutinib exposure. (B) Western blot showing decreased p-ERK protein expression in KYAE-1 tumour cell line (MYC and ERBB2 amplified) and (C) TE8 cell line (MYC amplified) following ibrutinib exposure. (D) Western blots showing that ibrutinib exposure results in downregulation of p-MYC S62 and total CMYC in KYAE-1 (MYC and ERBB2 amplified) and (E) TE8 (MYC amplified) oesophageal tumour cell lines.

require orthogonal validation. We recommend that subsequent work based on the siRNA analysis incorporates the use of individual siRNAs designed to target a gene, CRISPR-Cas9-mediated gene targeting or small molecule inhibitors given the well-documented off-target effects associated with RNAi reagents. ${ }^{51}$ We are also mindful of the limitations of using tumour cell lines in terms of modelling the human disease and would recommend that future work relating to oesophageal cancer should be validated using patient-derived tumour organoids (PDTOs) and xenografts (PDXs). PDXs, where surgically resected tumour samples are engrafted directly into immunocompromised mice, offer many advantages over standard cell-line xenograft models, including the preservation of the molecular heterogeneity frequently observed in human oesophageal tumours, and represent a genomically characterised platform with which to analyse drug sensitivity in relation to driver mutations for the purposes of biomarker discovery. In addition to commercially available PDX collections, patient-derived in vitro and in vivo models have also been propagated through academic collaboration..$^{52}$ Going forward, it would be important to use such platforms to identify the dynamic response to ibrutinib in relevant oesophageal cancer PDTOs and PDXs as a route to identifying mechanismsof resistance to BTK inhibition that might emerge. Finally, candidate predictive biomarkers identified from drug screening should be evaluated in oesophageal tumour tissues within the context of prospective 
clinical studies utilising targeted therapies and correlated with clinical outcome.

\section{Supplementary tables}

All online supplementary tables have been made publicly available and can be accessed using the following link: https://doi. org/10.5281/zenodo.826575.

\section{MATERIALS AND METHODS Cell lines}

Tumour cell lines were obtained from the American Type Culture Collection, European Collection of Cell Cultures and Deutsche Sammlung von Mikroorganismen und Zellkulturen, and maintained as per the supplier's instructions. Short tandem repeats (STR) geno typing of 10 loci was performed using the GenePrint 10 system (Promega) and used to confirm the identity of cell lines prior to storage. At monthly intervals, mycoplasma testing was carried out using MycoAlert Mycoplasma Detection Kit (Lonza).

\section{Array comparative genomic hybridisation}

All cell line and tumours were subjected to aCGH (Breakthrough Breast Cancer Research Centre) 32K BAC array platform and profiled as previously described. ${ }^{27}$ siRNA screening and processing 17 oesophageal cancer cell lines were optimised for siRNA screening conditions by titrating cell number and transfection lipid. Each cell line was transfected with a plate-arrayed siRNA library targeting 714 kinases and kinase-related genes (Dharmacon in a 384-well plate format) as described in Campbell et al. ${ }^{26}$

\section{Association testing}

An MP test was used to identify associations between specific features (histotype or driver gene mutation) and sensitivity to specific siRNAs. For each kinase we compared the observed difference between the median $\mathrm{Z}$ score of the interest group (mutant, tissue of interest or other grouping variable) and the median Z score of the 'other' group (wild-type, non-tissue of interest or inverse of other grouping variable) to that expected based on random permutation.

\section{Small molecule kinase inhibitor growth assays}

The compound screens comprised an in-house library of 80 small molecule inhibitors $(0.5,1,5,10,50,100,500$ and $1000 \mathrm{nM})$. Five hundred cells were seeded in 384-well plates. Twenty-four hours post seeding, drug treatment was initiated and cells were continuously exposed to the drugs (6 days), at which point cell viability was estimated using CellTiter-Glo (Promega). Luminescence values were normalised to the median of the per-plate dimethyl sulphoxide (DMSO) negative control wells and the dose-response relationships modelled using three-parameter logistic regression provided by the drc R package. AUC scores are represented as a proportion of the maximum area under curve (calculated as: $\log 10(500000)-\log 10(500)=3$ ).

\section{BTK and BTK-C PCR}

RNA was extracted from untreated cells using RNeasy Mini Kit (Qiagen). One microgram of RNA was converted to cDNA using reverse transcriptase with the iScript cDNA synthesis kit (BioRad) as per manufacturer's instructions. The cDNA was used to detect BTK or BTK-C with the following primers: BTK forward 5'-GGGGTTTGCTCAGACTGTCC-3', BTK-C forward 5'-AATGAAGGGGTCCTCCACCT-3' and BTK/BTK-C reverse 5'-AATCACTGCGGCCATAGCTT-3'. PCR was performed using Platinum Green Hot Start PCR 2x Master mix (Thermo Fisher) as per manufacturer's instructions.

\section{BTK and BTK-C real-time PCR}

RNA was extracted and converted to cDNA. Real time PCR was carried out using PowerUp SYBR Green Master mix (Thermo Fisher) and 20x PrimePCR primers for BTK and Actin (BioRad). For BTK-C, the primers used were those described by Eifert et al (Genes, Chromosomes and Cancer, 2013) and actin primers were forward 5'-CCCTGGCACCCAGCAC-3' and reverse 5'-GCCGATCCACACGGAGTAC-3'.

\section{Reverse transfections and cell viability assay}

Fifteen microlitres of each siRNA species were mixed with $750 \mu \mathrm{L}$ of optimum and incubated at RT $(10 \mathrm{~min})$. Fifty microlitres of RNAiMax (Invitrogen) were incubated with $2.5 \mathrm{~mL}$ optimum (10 $\mathrm{min})$. Two hundred fifty microlitres of transfection mix were added to the siRNA mix and incubated at RT (30 min). Of the transfection/siRNA mix, $17.5 \mu \mathrm{L}$ was plated in triplicate in 96-well plate format, then $80 \mu \mathrm{L}$ cells/well $\left(0.025 \times 10^{6}\right.$ cells/ $\mathrm{mL}$ ) were then seeded. After 5 days, $35 \mu \mathrm{L}$ CellTiter-Glo was added per well. The de-convoluted siRNA target sequences used are as follows:

siMYC1 AACGUUAGCUUCACCAACA siMYC2 GGAACUAUGACCUCGACUA SiMYC3 GAACACACAACGUCUUGGA siMYC4 CUACCAGGCUGCGCGCAAA siBTK1 GCUAUGGGCUGCCAAAUUU SiBTK2 GAAAGCAACUUACCAUGGU siBTK3 GGUAAACGAUCAAGGAGUU siBTKC1 GGUUAUUG GAUGCCCAUUAUU SiBTKC2 GGGAGAGCACACAGGU GAAUU

Table 2 Genetic dependencies in oesophageal cancer: Chong et al

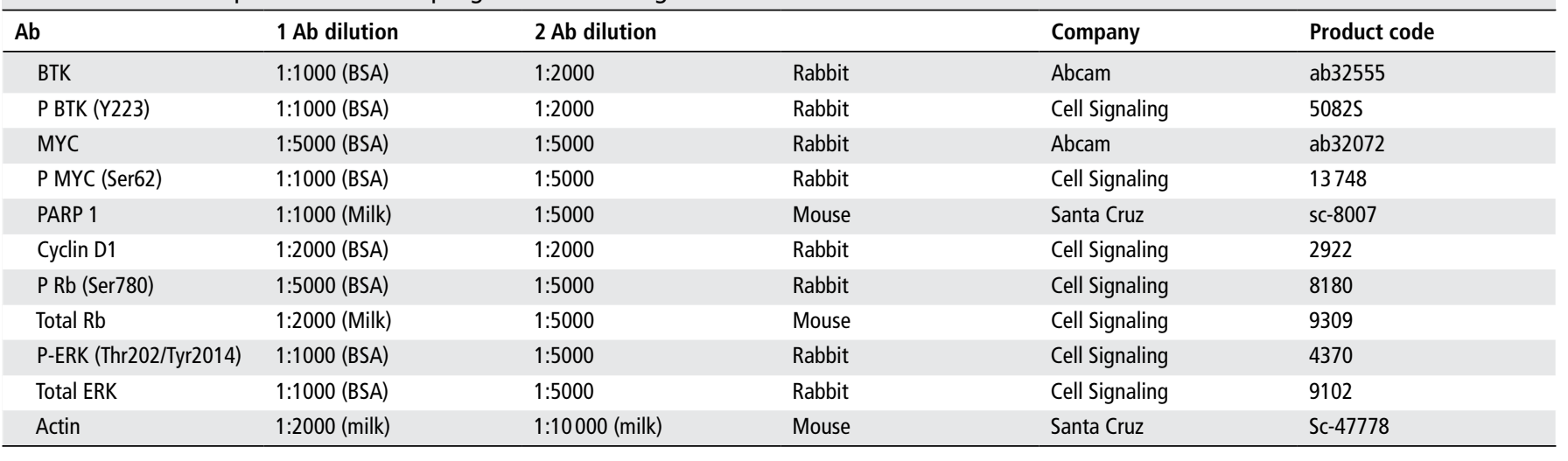




\section{Apoptosis assay}

Apoptotic cells were identified using an FITC Annexin V Kit (BD Bioscience). Cells were plated and treated the following day with ibrutinib for 48 or 72 hours, trypsinised, washed in phosphate-buffered saline (PBS) and resuspended in 1x Annexin binding buffer. Five microlitres of FITC Annexin V and $5 \mu \mathrm{L}$ propidium iodide (PI) and $400 \mu \mathrm{L}$ of $1 \mathrm{x}$ Annexin binding buffer were added. Data were analysed on a BD LSRII flow cytometer.

\section{Cell cycle assay}

Cells were plated and treated the following day with ibrutinib for 24 or 48 hours. Cells were trypsinised, washed in PBS and fixed in $70 \%$ ethanol, then treated with $50 \mu \mathrm{g} / \mathrm{mL}$ RNase A. PI was added $(50 \mu \mathrm{g} / \mathrm{mL})$ and data were analysed on a BD LSRII flow cytometer.

\section{Western blotting}

Cell lysates were separated using 4\%-12\%sodium dodecyl sulfate-polyacrylamide gel electrophoresis (SDS-PAGE) Bis-Tris gel, transferred to nitrocellulose membrane, blocked in 5\% milk and blotted with antibodies (table 2).

\section{BTK overexpression in MYC amplified oesophageal cancer cell lines}

BTK was overexpressed using a lentiviral vector containing the BTK ORF (Source Bioscience). Five micrograms of this plasmid with $4 \mu \mathrm{g}$ psPAX2 packaging plasmid and $1.5 \mu \mathrm{g}$ pMD2.G envelope plasmid were combined with Lipofectamine 3000 and P3000 (Thermo Fisher) and used to transfect $2 \times 10^{6} 293 \mathrm{~T}$ cells.

\begin{abstract}
Acknowledgements This work was funded by a Cancer Research UK programme grant to CJL (grant number C347/A8363) and Janssen and Pharmacyclics Pharmaceuticals as part of a sponsored research agreement with IYC. AA is funded by a Department of Defence grant. We acknowledge NHS funding to the NIHR Royal Marsden Hospital Biomedical Research Centre. We thank the Thornton Foundation, Cadogan Foundation and the Royal Marsden Cancer Charity for their financial support.
\end{abstract}

Contributors Data generation and analysis: IYC, LA, HB, AG, JC, RE, AR, MBL, PW, CJL. Data interpretation: IYC, LA, HB, SP, JSRF. Obtained funding: IYC, DC, IC, AA, CJL. Manuscript drafting: IYC, LA, SP, MD, IC, DC, AA, CJL. Approval of final manuscript: All authors.

Disclaimer IYC has received research funding from Janssen and Pharmacyclics as part of a sponsored research agreement. DC accepts research funding from the following institutions: Amgen, AstraZeneca, Bayer, Celgene, Merrimack, Medimmune, Merck Serono and Sanofi Oncology. IC is on the Advisory Board for Sanofi Oncology, Eli Lilly, Bristol Myers Squibb, MSD, Roche and Five Prime Therapeutics, accepts research funding from Janssen-Cilag, Sanofi Oncology, Merck Serono and Novartis, and has received honorarium from Taiho, Pfizer, Amgen, Eli Lilly and Gilead Science.

Competing interests IYC has received research funding from Janssen and Pharmacyclics as part of a sponsored research agreement. IC accepts research funding from Janssen-Cilag.

Provenance and peer review Not commissioned; externally peer reviewed.

Data sharing statement There are no additional unpublished data from this study available.

Open access This is an open access article distributed in accordance with the Creative Commons Attribution Non Commercial (CC BY-NC 4.0) license, which permits others to distribute, remix, adapt, build upon this work non-commercially, and license their derivative works on different terms, provided the original work is properly cited and the use is non-commercial. See: http://creativecommons.org/ licenses/by-nc/4.0/

(c) Article author(s) (or their employer(s) unless otherwise stated in the text of the article) 2018. All rights reserved. No commercial use is permitted unless otherwise expressly granted.

\section{REFERENCES}

1 Siegel RL, Miller KD, Jemal A. Cancer statistics. CA Cancer J Clin 2016;66:7-30.

2 Rustgi AK, El-Serag HB. Esophageal carcinoma. N Engl J Med 2014;371:2499-509.
3 Cancer Genome Atlas Research N, Analysis Working Group: Asan U, Agency BCC, Brigham, Women's H, Broad I et al Integrated genomic characterization of oesophageal carcinoma Nature 2017;541:169-75.

4 Gregson EM, Bornschein J, Fitzgerald RC. Genetic progression of Barrett's oesophagus to oesophageal adenocarcinoma. Br J Cancer 2016;115:403-10.

5 Siegel R, Naishadham D, Jemal A, et al. Cancer statistics, 2012. CA Cancer J Clin 2012;62:10-29.

6 Shah MA. Update on metastatic gastric and esophageal cancers. J Clin Oncol 2015;33:1760-9.

7 Cunningham D, Allum WH, Stenning SP, et al. Perioperative chemotherapy versus surgery alone for resectable gastroesophageal cancer. N Engl I Med 2006;355:11-20.

8 Cunningham D, Starling N, Rao S, et al. Capecitabine and oxaliplatin for advanced esophagogastric cancer. N Eng/ J Med 2008;358:36-46.

9 Van Cutsem E, Moiseyenko VM, Tjulandin S, et al. Phase III study of docetaxel and cisplatin plus fluorouracil compared with cisplatin and fluorouracil as first-line therapy for advanced gastric cancer: a report of the V325 Study Group. J Clin Oncol 2006; $24: 4991-7$

10 Guimbaud R, Louvet C, Ries P, et al. Prospective, randomized, multicenter, phase III study of fluorouracil, leucovorin, and irinotecan versus epirubicin, cisplatin, and capecitabine in advanced gastric adenocarcinoma: a French intergroup (Fédération Francophone de Cancérologie Digestive, Fédération Nationale des Centres de Lutte Contre le Cancer, and Groupe Coopérateur Multidisciplinaire en Oncologie) study. $J$ Clin Oncol 2014:32:3520-6.

11 Al-Batran SE, Hartmann JT, Probst S, et al. Phase III trial in metastatic gastroesophageal adenocarcinoma with fluorouracil, leucovorin plus either oxaliplatin or cisplatin: a study of the Arbeitsgemeinschaft Internistische Onkologie. J Clin Oncol 2008:26:1435-42.

12 Pozzo C, Barone C, Szanto J, et al. Irinotecan in combination with 5-fluorouracil and folinic acid or with cisplatin in patients with advanced gastric or esophagealgastric junction adenocarcinoma: results of a randomized phase II study. Ann Oncol 2004;15:1773-81.

13 Bouché $\mathrm{O}$, Raoul JL, Bonnetain F, et al. Randomized multicenter phase II trial of a biweekly regimen of fluorouracil and leucovorin (LV5FU2), LV5FU2 plus cisplatin, or LV5FU2 plus irinotecan in patients with previously untreated metastatic gastric cancer: a Federation Francophone de Cancerologie Digestive Group Study--FFCD 9803. J Clin Oncol 2004:22:4319-28

14 Roth AD, Fazio N, Stupp R, et al. Docetaxel, cisplatin, and fluorouracil; docetaxel and cisplatin; and epirubicin, cisplatin, and fluorouracil as systemic treatment for advanced gastric carcinoma: a randomized phase II trial of the Swiss Group for clinical Cancer research. J Clin Oncol 2007;25:3217-23.

15 Wilke H, Muro K, Van Cutsem E, et al. Ramucirumab plus paclitaxel versus placebo plus paclitaxel in patients with previously treated advanced gastric or gastrooesophageal junction adenocarcinoma (RAINBOW): a double-blind, randomised phase 3 trial. Lancet Oncol 2014;15:1224-35.

16 Fuchs CS, Tomasek J, Yong CJ, et al. Ramucirumab Monotherapy for previously treated advanced gastric or gastro-oesophageal junction adenocarcinoma (REGARD): an international, randomised, multicentre, placebo-controlled, phase 3 trial. Lancet 2014;383:31-9.

17 Bang YJ, Van Cutsem E, Feyereislova A, et al. Trastuzumab in combination with chemotherapy versus chemotherapy alone for treatment of HER2-positive advanced gastric or gastro-oesophageal junction Cancer (ToGA): a phase 3, open-label, randomised controlled trial. Lancet 2010;376:687-97.

18 Ross-Innes CS, Becq J, Warren A, et al. Whole-genome sequencing provides new insights into the clonal architecture of Barrett's esophagus and esophageal adenocarcinoma. Nat Genet 2015;47:1038-46.

19 Dulak AM, Stojanov P, Peng S, et al. Exome and whole-genome sequencing of esophageal adenocarcinoma identifies recurrent driver events and mutational complexity. Nat Genet 2013:45:478-86.

20 Stachler MD, Taylor-Weiner A, Peng S, et al. Paired exome analysis of Barrett's esophagus and adenocarcinoma. Nat Genet 2015;47:1047-55.

21 Secrier M, Li X, de Silva N, et al. Mutational signatures in esophageal adenocarcinoma define etiologically distinct subgroups with therapeutic relevance. Nat Genet 2016;48:1131-41.

22 Sawada G, Niida A, Uchi R, et al. Genomic Landscape of Esophageal Squamous Cell Carcinoma in a Japanese Population. Gastroenterology 2016;150:1171-82.

23 Song Y, Li L, Ou Y, et al. Identification of genomic alterations in oesophageal squamous cell Cancer. Nature 2014;509:91-5.

24 Chong IY, Cunningham D, Barber LJ, et al. The genomic landscape of oesophagogastric junctional adenocarcinoma. J Pathol 2013;231:301-10.

25 Mimura K, Kono K, Hanawa M, et al. Frequencies of HER-2/neu expression and gene amplification in patients with oesophageal squamous cell carcinoma. Br J Cancer 2005;92:1253-60.

26 Campbell J, Ryan CJ, Brough R, et al. Large-Scale profiling of kinase dependencies in Cancer cell lines. Cell Rep 2016:14:2490-501.

27 Turner N, Lambros MB, Horlings HM, et al. Integrative molecular profiling of triple negative breast cancers identifies amplicon drivers and potential therapeutic targets. Oncogene 2010;29:2013-23. 
28 Dulak AM, Schumacher SE, van Lieshout J, et al. Gastrointestinal adenocarcinomas of the esophagus, stomach, and Colon exhibit distinct patterns of genome instability and oncogenesis. Cancer Res 2012;72:4383-93.

29 Dulak AM, Stojanov P, Peng S, et al. Exome and whole-genome sequencing of esophageal adenocarcinoma identifies recurrent driver events and mutational complexity. Nat Genet 2013;45:478-86.

30 Agrawal N, Jiao Y, Bettegowda C, et al. Comparative genomic analysis of esophageal adenocarcinoma and squamous cell carcinoma. Cancer Discov 2012;2:899-905.

31 Beroukhim R, Mermel CH, Porter D, et al. The landscape of somatic copy-number alteration across human cancers. Nature 2010;463:899-905.

32 Bandla S, Pennathur A, Luketich JD, et al. Comparative genomics of esophageal adenocarcinoma and squamous cell carcinoma. Ann Thorac Surg 2012;93:1101-6.

$33 \mathrm{Hu} \mathrm{N}$, Clifford RJ, Yang HH, et al. Genome wide analysis of DNA copy number neutral loss of heterozygosity (CNNLOH) and its relation to gene expression in esophageal squamous cell carcinoma. BMC Genomics 2010;11:576.

34 Goh XY, Rees JR, Paterson AL, et al. Integrative analysis of array-comparative genomic hybridisation and matched gene expression profiling data reveals novel genes with prognostic significance in oesophageal adenocarcinoma. Gut 2011;60:1317-26.

35 Dutton SJ, Ferry DR, Blazeby JM, et al. Gefitinib for oesophageal cancer progressing after chemotherapy (COG): a phase 3, multicentre, double-blind, placebo-controlled randomised trial. Lancet Oncol 2014;15:894-904.

36 Marshall CJ. Specificity of receptor tyrosine kinase signaling: transient versus sustained extracellular signal-regulated kinase activation. Cell 1995;80:179-85.

37 Hendriks RW, Yuvaraj S, Kil LP. Targeting Bruton's tyrosine kinase in B cell malignancies. Nat Rev Cancer 2014;14:219-32.

38 Aoki Y, Isselbacher KJ, Pillai S. Bruton tyrosine kinase is tyrosine phosphorylated and activated in pre-B lymphocytes and receptor-ligated B cells. Proc Natl Acad Sci U S A 1994;91:10606-9.

39 Eifert C, Wang X, Kokabee L, et al. A novel isoform of the B cell tyrosine kinase BTK protects breast Cancer cells from apoptosis. Genes Chromosomes Cancer 2013;52:961-75.
40 Kokabee L, Wang X, Sevinsky CJ, et al. Bruton's tyrosine kinase is a potential therapeutic target in prostate cancer. Cancer Biol Ther 2015;16:1604-15.

41 Grassilli E, Pisano F, Cialdella A, et al. A novel oncogenic BTK isoform is overexpressed in Colon cancers and required for RAS-mediated transformation. Oncogene 2016;35:4368-78

42 Wang JD, Chen XY, Ji KW, et al. Targeting btk with ibrutinib inhibit gastric carcinoma cells growth. Am J Trans/ Res 2016;8:3003-12.

43 Byrd JC, Furman RR, Coutre SE, et al. Targeting BTK with ibrutinib in relapsed chronic lymphocytic leukemia. N Engl J Med 2013;369:32-42.

44 Wang ML, Rule S, Martin P, et al. Targeting BTK with ibrutinib in relapsed or refractory mantle-cell lymphoma. N Engl J Med 2013;369:507-16.

45 Grabinski N, Ewald F. Ibrutinib (ImbruvicaTM) potently inhibits ErbB receptor phosphorylation and cell viability of ErbB2-positive breast cancer cells. Invest New Drugs 2014;32:1096-104.

46 Chen J, Kinoshita T, Sukbuntherng J, et al. Ibrutinib inhibits ERBB receptor tyrosine kinases and HER2-Amplified breast Cancer cell growth. Mol Cancer Ther 2016;15:2835-44.

47 Sears R, Leone G, DeGregori J, et al. Ras enhances Myc protein stability. Mol Cell 1999:3:169-79.

48 Sears R, Nuckolls F, Haura E, et al. Multiple Ras-dependent phosphorylation pathways regulate Myc protein stability. Genes Dev 2000;14:2501-14.

49 Tischler J, Lehner B, Fraser AG. Evolutionary plasticity of genetic interaction networks. Nat Genet 2008;40:390-1.

50 Treon SP, Tripsas CK, Meid K, et al. Ibrutinib in previously treated Waldenström's macroglobulinemia. N Eng/ J Med 2015;372:1430-40.

51 Jackson AL, Linsley PS. Recognizing and avoiding siRNA off-target effects for target identification and therapeutic application. Nat Rev Drug Discov 2010;9:57-67.

52 Pauli C, Hopkins BD, Prandi D, et al. Personalized In Vitro and In Vivo Cancer Models to Guide Precision Medicine. Cancer Discov 2017;7:462-77. 